\title{
ENTRENAMIENTO MENTAL EN KARATECAS: EFECTO DEL TIEMPO DE IMAGINACIÓN DE UNA KATA SOBRE EL NIVEL DE EJECUCIÓN
}

\author{
Ivonne Navarro Navarro, Gerardo Araya Vargas y Walter Salazar Rojas \\ Escuela de Educación Física y Deportes \\ Universidad de Costa Rica, San José, Costa Rica \\ E-mail:ivomana@hotmail.com
}

\begin{abstract}
Resumen
Navarro, I., Araya, G. y Salazar, W. (2002). Entrenamiento mental en karatecas: Efecto del tiempo de imaginación de una kata sobre el nivel de ejecución. Revista de Ciencias del Ejercicio y la Salud, 2(1), 5560. El objetivo de esta investigación fue determinar el efecto de la duración de una práctica mental, combinada con práctica física, sobre el nivel de ejecución de una kata. Participaron 15 varones, quienes eran avanzados; su edad promedio era de 21 años. Se realizaron dos sesiones, en las cuales los sujetos observaron a un modelo quien hizo una vez la kata (conjunto de movimientos -ataques y defensas-), e inmediatamente después ellos la ejecutaron una vez también. Al concluir estas sesiones, se llevó a cabo el pretest de la ejecución de la kata y se distribuyó a la población aleatoriamente en tres grupos. En cada sesión los tres grupos observaban al modelo que ejecutaba la kata (una vez), luego un grupo hacía una práctica mental de 8 minutos, en donde realizaban una repetición mental de la kata (GEM8M); en ese mismo momento otro grupo (GEM12M) hacía lo mismo; pero en 12 minutos, el grupo control (NPM) no tuvo práctica mental; después los tres grupos realizaban la práctica física, que consistía en la ejecución de la kata (una vez). La duración del tratamiento fue de 4 semanas, 2 veces por semana. Se llevaron a cabo 3 mediciones del nivel de ejecución de la kata, dichas calificaciones estuvieron a cargo de un juez avalado internacionalmente. La primera medición se hizo concluida las sesiones sin práctica mental (pretest), la segunda a la mitad del tratamiento (Test1) y la tercera al finalizar las sesiones de entrenamiento mental (Test2). Los resultados mostraron que no hubo diferencia entre el promedio de ejecución de la kata de los tres grupos en el pretest. Tampoco existió diferencia entre el GEM8M y el GEM12M en lo referente a las mediciones test1 y test2; pero sí existió diferencia significativa entre estos 2 grupos y el grupo NPM en cada una de estas mediciones. Además se encontró diferencia significativa entre las tres mediciones (pretest, test 1, test 2), para los tres grupos, lo que implica que los sujetos fueron progresando a lo largo del tratamiento, no obstante los grupos que tenían entrenamiento mental en su tratamiento mejoraron más que el grupo que tenía solo práctica física. PALABRAS CLAVES: Entrenamiento mental, kata, karate, práctica mental, imaginería.
\end{abstract}

\section{INTRODUCCIÓN}

Una tradicional creencia en el mundo del deporte es que la única manera de aprender una destreza motora es gastando largas y duras horas en la práctica física. Sin embargo leer sobre una destreza, ver un video o simular un movimiento también es efectivo cuando el objetivo es adquirir una destreza motora (Murphy y Jowdy, 1992).

Es por ello que la literatura reciente hace énfasis en la práctica mental y en cómo ésta puede afectar el deporte o el rendimiento motor. Entendiéndose como práctica mental aquella en donde se exige al deportista que imagine la práctica del movimiento específico que desea entrenar y mejorar sin que él mismo ejecute el movimiento (Eberspächer, 1995).

La literatura de la práctica mental ha generado dos grandes propuestas o enfoques para tratar de explicar los efectos de la práctica mental, uno de ellos es la hipótesis psiconeuromuscular, la cual está relacionada con la actividad muscular en la práctica mental, es decir que cuando una persona imagina que está moviendo alguna parte de su cuerpo, los músculos específicos asociados 
con el movimiento que está siendo imaginado, tienen variaciones en su actividad mioeléctrica (Weinberg y Gould, 1995). La segunda hipótesis es la del aprendizaje simbólico, la cual plantea que la práctica mental beneficia el desempeño de una destreza, ya que los sujetos se preparan cognitivamente para la actividad, como por ejemplo la preparación de un plan de ejecución de una tarea, en donde se puede incluir el ensayo de la secuencia de los aspectos relevantes de la misma, considerar la característica espacial de la destreza, aclarar las metas o analizar los posibles problemas en la ejecución de la tarea (Weinberg y Gould, 1995).

En relación a esta última hipótesis, Feltz y Landers (1983), realizaron un metaanálisis en el que se incluyeron 60 estudios en los que se aplicaba práctica mental. Encontraron un tamaño de efecto promedio total de 0.48, sugiriendo que la práctica mental es más efectiva que no practicar del todo una destreza, para mejorar su rendimiento. Su análisis también reveló un mayor tamaño de efecto para las tareas cognitivas, que para las tareas motoras o de resistencia.

Otro estudio desarrollado en está área es el de Greenspan y Feltz (1989), quienes revisaron 23 investigaciones publicadas acerca del efecto que tiene el trabajar diferentes factores psicológicos como son el estrés, imágenes mentales, relajación y refuerzos, en los resultados de una competencia. Los investigadores concluyeron que en general las intervenciones psicológicas mejoraron efectivamente, incluyendo imágenes mentales, el desempeño competitivo en atletas universitarias y adultos.

Meacci y Price (1985), estudiaron el efecto de 4 tipos de técnicas de aprendizaje para la ejecución de una destreza de golf, las cuales consistían en: 1) relajación, visualización, simulación del movimiento (ejecución de movimiento de tiro sin golpear la bola en realidad); 2) ejecución de la destreza (práctica física); 3) relajación, visualización, simulación y repetición de la destreza motora (combinación -físico y mental-); 4) grupo control el cual no realizó ninguna de las técnicas anteriores. Los resultados de ese estudio revelaron una diferencia significativa en el desarrollo del tiro de golf del pretest al postest entre el grupo combinado y los otros 3 grupos. Lo que indica que una combinación de práctica física y métodos cognitivos conducen a una mejor adquisición de la destreza que una práctica física sola, además todos los tres métodos de aprendizaje aumentaron significativamente la retención de la destreza aprendida.

Garza y Feltz (1998) estudiaron el efecto de una práctica mental en el desempeño, eficacia y autoconfianza en patinaje artístico, con 27 mujeres de edades de 10 a 18 años, miembros de la asociación de patinaje artístico de Estados Unidos quienes fueron asignados aleatoriamente a tres grupos. Un grupo hacía una práctica mental basada en el dibujo libre sobre papel (grupo A), para ello se les pidió a las patinadoras que se visualizaran en la pista de patinaje como si estuvieran en el escenario de competencia; después se puso la música de cada patinadora y se les pidió que dibujaran su propia rutina. Otro grupo realizaba una práctica mental la cual consistía en caminar por la pista diciendo palabras claves de su rutina en voz alta (grupo B) y el grupo control, hizo solamente un trabajo de estiramiento. Los tratamientos tuvieron una duración de 4 semanas. Se encontró, una mejora significativa en el grupo A y B tanto en su ejecución de saltos y giros como en la auto-confianza precompetencia y en su opinión de eficacia comparada con el control.

Andre y Means (1986) manipularon la velocidad de la imagen usada en la práctica mental. Ellos especularon que usar una imagen de movimiento lento en práctica mental podría aumentar la efectividad de la práctica, además al enriquecer la experiencia de imagen de los sujetos, se podría inducir a una tarea motora de forma más vívida y clara, lo que eventualmente mejoraría la ejecución.

Se trabajó con 3 grupos: un grupo de práctica mental normal (PM), un grupo con movimiento lento en la práctica mental (PML) y un grupo placebo. La hipótesis 
planteada por los investigadores fue que ambos grupos PM y PML mostrarían significativamente un mayor avance del pre al postratamiento que el grupo placebo. Además que la inclusión del factor de práctica mental con movimientos lentos conduciría al grupo de PML a tener un mayor nivel de ejecución que el grupo de PM y que el grupo placebo presentaría un aumento en la calidad de la ejecución, en un margen no significativo de acuerdo a las expectativas de los resultados.

La tarea escogida para la investigación fue el lanzamiento de un disco de frisbee (lanzamiento de frisbee a hoyos). Los sujetos observaron la demostración de la tarea y luego cada uno la practicó 5 veces.

Los 3 grupos recibieron una fase de relajación. La práctica mental del grupo PM consistió en 3 series de 3 repeticiones de la destreza a nivel mental; el grupo de PML fue esencialmente igual que el grupo anterior, sin embargo fue instruido para realizar la segunda y tercera ejecución de la tarea de cada serie con movimientos lentos, la primera ejecución mental de la tarea de cada serie fue descrita en velocidad normal, dándoles a los sujetos un punto de referencia natural para su imagen de movimiento lento. El grupo placebo recibió la misma cantidad de tiempo de práctica mental que los otros grupos; pero las instrucciones que recibieron no contenían la información específica de la tarea. Los resultados indican que los sujetos de PM mejoraron un promedio de 2.27 tiros del pre al postest (10.94\% de aumento); el grupo de PML incrementó un promedio de 1.4 tiros (5.62\% de aumento). En el grupo placebo su promedio de ejecución disminuyó 0.32 tiros (1.57\% de disminución). Los resultados indican que aunque hubo aparentemente alguna mejora en los dos grupos del tratamiento, este cambio no fue significativamente diferente al demostrado por el grupo placebo.

De acuerdo a la escala de chequeo que se les administró después de la prueba a los sujetos, la mayoría reportaron que la velocidad de sus imágenes fueron marginalmente más lentas que la velocidad de la vida real.

Es grande el número de investigaciones en donde se utiliza la práctica mental; sin embargo existe todavía mucha controversia en relación a las características de dicha práctica con base al tiempo, el tipo de imagen, velocidad de la imagen, al aspecto cognitivo-físico y a los estímulos que la práctica mental produce a nivel muscular y cerebral, además de la duda sobre la verdadera efectividad de la práctica mental.

En fin muchas preguntas $y$ problemas han surgido a través del tiempo en lo que a la práctica mental se refiere.

Dentro de los aspectos que deben ser aclarados cabe destacar el trabajo de Andre y Means (1986) quienes manipularon la velocidad de la imagen, sin embargo la velocidad de imagen en los sujetos no pudo ser lo suficientemente controlada por los investigadores, además el hecho de trabajar con una tarea clasificada como de un bajo componente cognitivo (lanzamiento de frisbee a hoyos), pudo afectar los resultados, ya que de acuerdo a muchos estudios basados en la teoría del aprendizaje simbólico (Feltz y Landers, 1983) la práctica mental es más efectiva en tareas con un mayor componente cognitivo que físico.

Se habla de la combinación de práctica física-mental y en lo que respecta a la parte física, áreas como la fisiología se han encargado de estudiar las respuestas y adaptaciones que se obtienen de acuerdo a la duración del ejercicio; sin embargo esta variable (tiempo) en la práctica mental es un tema que requiere todavía de mucha información y por lo tanto de mucha investigación.

Es por ello que se planteó este estudio, en donde se trabajó diferentes tiempos de práctica mental, con una tarea con alto componente cognitivo y además se aplicó un tratamiento más largo, en relación a la duración de los tratamientos aplicados en los estudios revisados.

Estableciendo como objetivo en la presente investigación, determinar el efecto de la duración de la práctica mental, combinada con práctica física, sobre el nivel de ejecución de una kata. 


\section{METODOLOGÍA}

\section{Sujetos}

En el estudio participaron 15 sujetos voluntarios. Todos ellos de sexo masculino con una edad entre 18 y 26 años, practicantes del arte marcial karate, quienes se ubicaban en el mismo nivel técnico, esto con base al color de la cinta (verde, morada). Los sujetos entrenaban una hora $\mathrm{y}$ media, con una frecuencia de 2 veces por semana.

\section{Instrumentos}

Se utilizó un equipo de audio y una grabación de la secuencia de movimientos de la kata, la cual era una kata avanzada, con respecto al nivel de los sujetos, en la disciplina de Artes Marciales Karate-Do (Shoto-Kan). Un modelo (cinta negra) quien ejecutaba la kata y un árbitro de karate.

\section{Procedimientos}

Antes de iniciar el tratamiento se realizaron dos sesiones, cada una de ellas al inicio de la sesión de entrenamiento regular de karate, en dichas sesiones los sujetos observaron al modelo quien hizo una vez la kata del estudio, la cual era desconocida por los sujetos, luego todos ejecutaron una vez la kata, siguiendo al modelo. Al concluir estas sesiones, se llevó cabo la primera medición, en donde se evaluaba la secuencia y calidad de ejecución de la kata y se prosiguió a distribuir a la población aleatoriamente en tres grupos.

En cada sesión los tres grupos observaban al modelo que ejecutaba la kata 1 vez, luego el grupo (GEM8M) guiados por una grabación de audio de la secuencia de la kata, hacía una práctica mental por 8 minutos, en donde realizaba una repetición mental de la kata; al mismo tiempo el grupo (GEM12M) hacía lo mismo; pero en 12 minutos, el grupo NPM no tuvo práctica mental; después todos realizaban la parte física , que consistía en la ejecución de la kata, una vez.
La duración del tratamiento fue de 4 semanas, con una frecuencia de 2 veces por semana, a parte de las 2 sesiones de familiarización con la kata (antes del pretest).

Se hicieron 3 mediciones del nivel de ejecución de la kata (calificaciones), las cuales estaban a cargo por un profesional avalado por la Asociación Costarricense de arbitraje de Karate, siguiendo estándares oficiales internacionales. La primera medición (pretest) se hizo concluidas las sesiones sin práctica mental, la segunda (Test1) a la mitad del tratamiento (al final de la cuarta sesión), y la tercera (Test2) al finalizar las sesiones de entrenamiento mental (final de la octava sesión).

\section{Análisis estadístico}

Con los datos obtenidos se procedió a obtener la estadística descriptiva (promedio y desviaciones estándar) por grupos y por mediciones; además de la estadística inferencial, para lo que se realizó un análisis de varianza de dos vías $3 \times 3$ (grupos $\mathrm{X}$ mediciones) y el correspondiente análisis de efectos simples, cuando fue necesario.

\section{RESULTADOS}

Como se puede apreciar en el cuadro 1 , los resultados de los valores promedios de las mediciones del GEM8M y GEM12M son iguales; sin embargo se realizó un análisis de varianza para ver si existía diferencia significativa entre los grupos experimentales y los puntajes del grupo control, entre mediciones. Estos resultados se presentan en el cuadro 2.

Cuadro 1. Promedio y desviación estándar del nivel de ejecución de la kata por grupo en cada medición.

\begin{tabular}{lccc}
\hline Grupo & Pretest & Test1 & Test2 \\
\hline GEM8M & $3 \pm 0.89$ & $6.40 \pm 0.89$ & $8.40 \pm 0.89$ \\
GEM12M & $3 \pm 1.22$ & $6.40 \pm 0.55$ & $8.40 \pm 1.14$ \\
NPM & $2 \pm 0.71$ & $4 \pm 1$ & $5.20 \pm 0.84$ \\
\hline
\end{tabular}


Cuadro 2. Resultado del ANOVA 3 X 3 (grupos $x$ mediciones) para los puntajes del nivel de ejecución de la kata

\begin{tabular}{lcc}
\hline Fuente & $\mathbf{F}$ & $\boldsymbol{\omega}^{2}$ \\
\hline Grupo & $13.52^{*}$ & 0.17 \\
Mediciones & $198.74^{*}$ & 0.65 \\
Interacción & $9.68^{*}$ & 0.03 \\
\hline
\end{tabular}

$* p<0.05$

Se encontró diferencias significativas entre grupos $(F: 13.52 ; p<0.05)$ y entre mediciones $(F: 198.74 ; p<0.05)$ y además se encontró interacción significativa entre grupos y mediciones $(F: 9.68 ; p<0.05)$.

Sin embargo el efecto de las mediciones fue más importante teniendo un $65 \%$ de la varianza explicada $\left(\omega^{2}=0.65\right)$ de la variable dependiente. En total, solo un 15\% correspondió a varianza de error. El gráfico 1 ilustra la interacción.

Gráfico 1. Interacción significativa de grupos por mediciones para los puntajes del nivel de ejecución de la kata.

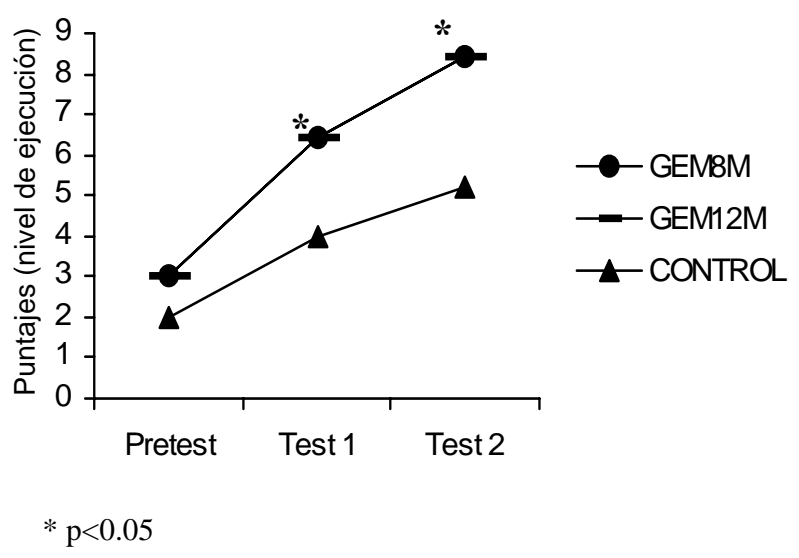

El análisis de efectos simples, aplicado, mostró que no hubo diferencia entre el promedio de ejecución de la kata de los tres grupos en el pretest.

En lo que respecta a las mediciones test1 y test2 no existió diferencia significativa entre el GEM8M y el GEM12M, pero sí existió diferencia significativa entre estos dos grupos y el grupo NPM en cada una de estas mediciones.
Por otra parte, se encontró una diferencia significativa entre las tres mediciones (pretest, Test1, Test2), para los tres grupos, lo que implica que los sujetos fueron progresando a lo largo del tratamiento, en los tres grupos, no obstante los grupos que tenían entrenamiento mental mejoraron más que el grupo que tenía solo práctica física.

\section{DISCUSIÓN}

Está investigación tenía como objetivo determinar el efecto de dos tipos de práctica mental de diferente duración combinada con práctica física, sobre el nivel de ejecución de una kata.

Los resultados obtenidos en este estudio confirman lo expuesto por Meacci y Price (1985) los cuales encontraron que una combinación de práctica física y mental conduce a una mejor adquisición de una destreza.

No obstante cuando se refiere a la duración del entrenamiento mental, la cual fue la variable independiente del estudio, no se logró demostrar un impacto significativo, sobre el nivel de ejecución, relativo al tiempo empleado en imaginar la secuencia de movimientos de la kata. Cabe acotar que los dos grupos de práctica mental, repetían mentalmente la kata, guiados por una grabación de audio, pero un grupo imaginaba la kata cuatro minutos más que el otro, es decir la velocidad de su práctica mental era menor. Por lo tanto, independientemente del tiempo y velocidad requerida para imaginar la kata, el hecho de realizar mentalmente la secuencia de los movimientos y luego ejecutarlos físicamente, produjo mayores mejoras en el nivel de ejecución en comparación con el grupo que solo hacía práctica física (Gráfico 1).

Además es importante resaltar que en esta investigación, se consideró el componente cognitivo de la tarea, tomando en cuenta lo demostrado por el meta-análisis de Feltz y Landers (1983) en relación con una mayor efectividad de la práctica mental en las tareas que tienen un alto componente cognitivo, como es el caso de la kata. 
Estos resultados confirman lo encontrado por Andre y Means (1986), quienes tampoco hallaron diferencia significativa en el rendimiento (prueba de precisión) con respecto a la duración de la secuencia mental. No obstante, la destreza empleada por estos investigadores no tenía tan alto componente cognitivo como la destreza (kata) empleada en este estudio.

Concluyendo con base en la información obtenida, que al combinar la práctica física y mental se obtienen mejores resultados en la destreza que se está aprendiendo; si se compara con la práctica meramente física. Sin embargo, parece que el tiempo de práctica mental no está directamente relacionado con un mayor rendimiento físico. No obstante, si la diferencia entre los tiempos de práctica mental se ampliara, tal vez así, se podría ver alguna diferencia en su impacto en el rendimiento.

\section{REFERENCIAS}

Andre, J. C. y Means, J. (1986). Rate of imagery in mental practice: An experimental investigation. Journal of Sport Psychology, 8, 124-128.

Eberspächer, H. (1995). Entrenamiento mental. Un manual para entrenadores $y$ deportistas. Barcelona: INDE.

Feltz, D. y Landers, D. (1983). The effects of mental practice on motor skill learning and performance: A meta-analysis. Journal of Sport Psychology, 5, 25-57.

Garza, D. y Feltz, D. (1998). Effects of selected mental practice on performance, self-efficacy, and competition confidence of figure skaters. The Sport Psychologist, 12, 1-15.

Greespan, M. J. y Feltz, D. F. (1989). Psychological interventions with athletes in competitive situations. The Sport Psychologist, 3, 219-236.

Meacci, W. y Price, E. (1985). Acquisition and retention in golf putting skill through the relaxation, visualization and body rehearsal intervention. Research Quartely for Exercise and Sport, 56, 176-179.

Murphy, S. y Jowdy, D. (1992). Imagery and mental practice. In T. Horn (Ed.), Advances is sport and exercise psychology. Champaign, IL: Human Kinetics.

Weinberg R. y Gould D. (1995). Foundations of Sport and Exercise Psychology. Champaign, IL: Human Kinetics. 